\title{
Effects of impurity seeding in DIII-D radiating mantle discharges
}

\author{
G.L. Jackson, M. Murakami ${ }^{1}$, G.R. McKee $^{2}$, D.R. Baker, \\ J.A. Boedo ${ }^{3}$, R.J. La Haye, C.J. Lasnier ${ }^{4}$, A.W. Leonard, \\ A.M. Messiaen ${ }^{5}$, J. Ongena ${ }^{5}$, G.M. Staebler, B. Unterberg ${ }^{6}$, \\ M.R. Wade ${ }^{1}$, J.G. Watkins ${ }^{7}$, W.P. West \\ General Atomics, San Diego, California, United States of America \\ ${ }^{1}$ Oak Ridge National Laboratory, Oak Ridge, Tennessee, USA \\ ${ }^{2}$ University of Wisconsin, Madison, Wisconsin, USA \\ ${ }^{3}$ University of California, San Diego, California, USA \\ ${ }^{4}$ Lawrence Livermore National Laboratory, Livermore, California, USA \\ ${ }^{5}$ Plasma Physics Laboratory, Association Euratom, Ecole Royale Militaire/Koninklijke \\ Militaire School, Brussels, Belgium \\ ${ }^{6}$ Forschungszentrum Jülich GmbH, Euratom Association, Jülich, Germany \\ ${ }^{7}$ Sandia National Laboratories, Albuquerque, New Mexico, USA \\ E-mail: jackson@legacy.gat.com
}

Received 29 May 2000, accepted for publication 20 June 2001

Published 30 January 2002

Online at stacks.iop.org/NF/42/28

\begin{abstract}
Impurity injection with noble gases has been used in DIII-D to increase radiation in the mantle region, with confinement enhancements above the ITERL-89P L mode scaling relation in both diverted and limited discharges. For discharges with an $\mathrm{L}$ mode edge, impurity injection produces a prompt increase in confinement and a more gradual increase in density. These changes occur at densities and radiated power fractions significantly lower than those observed in the TEXTOR tokamak device. ELMing H mode discharges with active pumping and high deuterium gas feed (puff and pump) exhibit an increase in density with no degradation in energy confinement after impurity injection, increasing to nearly the Greenwald density limit following a spontaneous transition several hundred milliseconds after impurity injection. The highest density phase of both $\mathrm{L}$ mode and ELMing $\mathrm{H}$ mode radiating mantle discharges is usually terminated after the onset of $n=2$ MHD activity, identified as an $m / n=3 / 2$ neoclassical tearing mode. A reduction in density fluctuations after impurity injection in the mantle region has been measured using beam emission spectroscopy in L mode discharges and is coincident with reductions in thermal diffusivity and increases in core toroidal rotation. The similarities and differences between these types of impurity seeded discharge will be presented.
\end{abstract}

PACS numbers: $52.55 . \mathrm{Fa}, 52.25 . \mathrm{Vy}$

\section{Introduction}

Intrinsic impurities in tokamak discharges, namely carbon, oxygen and medium or high $Z$ metals, have generally been regarded as deleterious to sustained high performance and reproducible discharges. However, non-intrinsic impurities have been shown, under some conditions, to produce confinement improvements above L mode [1] scaling, and the radiating mantle which can accompany these impurities can minimize the peak heat flux to plasma facing components: an important design consideration for fusion ignition devices. We report in this article on a series of experiments in DIII-D to explore the role of non-intrinsic impurity gases, i.e. neon and argon, in DIII-D discharges with confinement enhancements above L mode.

Several tokamaks have previously reported confinement enhancements above $\mathrm{L}$ mode with injection of neon or other impurities [2-10]. The first such studies were for $\mathrm{Z}$ mode on ISX-B [2], and the effect has been most extensively documented in the TEXTOR tokamak [3,11-13]. The TEXTOR group has developed a simple scaling for their radiative improved (RI) mode discharges [3], namely

$$
\tau_{R I-\text { mode }} / \tau_{\text {ITERH-93P }}=c_{1} \bar{n}_{e} / n_{G W}
$$

where the constant $c_{1}$ is approximately unity, $\tau_{I T E R H-93 P}$ is the ELM-free $\mathrm{H}$ mode confinement scaling relation [14], 
and $n_{G W}$ the Greenwald density limit [15] is defined as $n_{G W}\left(10^{20} \mathrm{~m}^{-3}\right)=I_{p}(\mathrm{MA}) /\left(\pi a(\mathrm{~m})^{2}\right)$. Both ISX-B and TEXTOR had a nearly circular cross-section, a poloidal limiter and an L mode edge. Non-intrinsic impurities have also been injected into diverted devices with an $\mathrm{H}$ mode edge in ASDEX Upgrade, JFT-2M, JET and DIII-D (prior to the experiments described here). Confinement enhancements above L mode were obtained in all these devices, but often there was a confinement degradation when compared with $\mathrm{H}$ mode scaling relations, particularly at the highest densities $[8,10]$. Although the effect of improved confinement with impurity seeding has been observed and well documented on many devices, the underlying physical mechanisms responsible for this improvement have not been well understood.

The goals of the experiments reported here are:

(a) To obtain enhanced confinement discharges in DIII-D with a large fraction of radiated power in the mantle region;

(b) To extend the results of TEXTOR to a larger machine, providing size scaling;

(c) To explore the parameter range in which these impurity seeded discharges with enhanced confinement can be obtained;

(d) To evaluate the physical mechanisms leading to improved confinement with impurity seeding.

In this article we will describe impurity seeded discharges with enhanced confinement, defined as confinement above L mode scaling. As discussed below, the degree of confinement enhancement is a function of many variables and we will quantify this for the various types of discharge presented. To represent the data in this article, we compare measured confinement values with either the ITERL-89P L mode [1] or the ITERH-93P ELM-free H mode [14] scaling relations. We will sometimes apply the ITERH-93P ELM-free scaling relation to $\mathrm{L}$ mode discharges with impurity seeding because this will allow comparison with other devices which have used this scaling in the literature.

DIII-D discharges with non-intrinsic radiation have been obtained under a variety of conditions and in various configurations. In this article we will discuss two types of discharge:

(a) lower single null (LSN) puff and pump ELMing H mode,

(b) L mode (either an inner wall limited (IWL) divertor or an upper single null (USN) divertor).

The $\mathrm{L}$ mode confinement enhancement factor is defined as $H_{89 P}=\tau_{E} / \tau_{\text {ITERL-89P }}$ and similarly the $\mathrm{H}$ mode confinement enhancement factor is $H_{93 P}=\tau_{E} / \tau_{\text {ITERH-93P. }}$. In all the discharges discussed in this article, the $\nabla|B| \times B$ drift direction is towards the floor, for example, the drift is away from the $\mathrm{X}$ point in a USN divertor configuration. The temporal evolution in normalized density and normalized confinement space, typical of the discharges discussed in the following sections, is shown in Fig. 1.

We will focus on discharges in which impurity injection leads to increased radiation in the mantle region and improved confinement in the core. The pathological case where the core temperature cools and impurity radiation peaks on-axis is not discussed here. Impurity seeded discharges with high

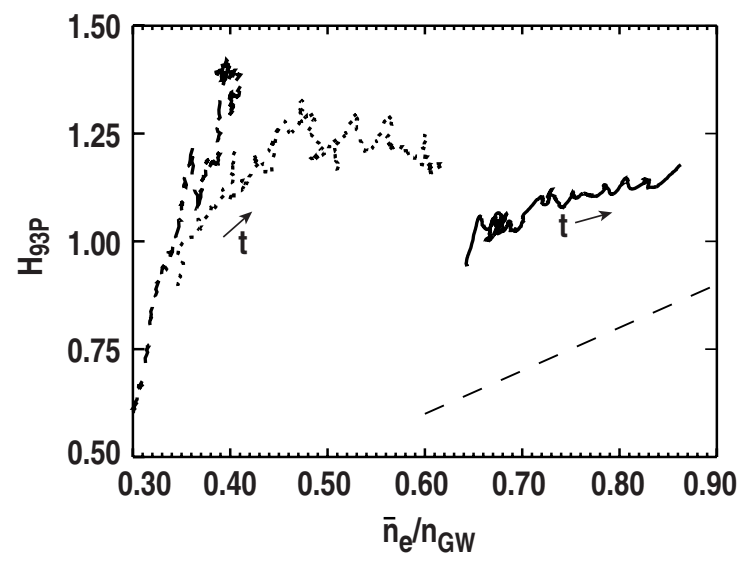

Figure 1. Phase space diagram of the temporal evolution typical of the types discussed in this article for three impurity seeded discharges during the impurity puffing phase: puff and pump ELMing $\mathrm{H}$ mode (solid curve), diverted with an L mode edge (dashed curve on left) and inner wall limited (dotted curve). TEXTOR RI mode scaling was used, Eq. (1) being shown as a dashed line (lower right).

deuterium flow where impurity radiation is primarily in the divertor region have been reported previously [16] but, except for comparisons, they are not presented here. Throughout this article we define the mantle region of a discharge as $0.5 \leqslant \rho \leqslant 1.0$ and the core region as $\rho<0.5$, where $\rho$ is the normalized radius. We define the fraction of core, mantle, divertor and total radiated power as $\gamma_{\text {core }}=P_{\text {core }} / P_{\text {in }}$, $\gamma_{\text {mantle }}=P_{\text {mantle }} / P_{\text {in }}, \gamma_{d i v}=P_{\text {div }} / P_{\text {in }}$ and $\gamma_{\text {tot }}=P_{\text {tot }} / P_{\text {in }}$, respectively. No RF heating was used for the discharges described so the input power is $P_{i n}=P_{N B}+P_{\text {ohmic }}$ where $P_{N B}$ and $P_{\text {ohmic }}$ are the neutral beam and ohmic heating powers, respectively.

In addition to the two types of discharge described above, a third category of discharges, double null divertor (DND) high performance with $H_{89 P}>2$, have also been achieved in DIII-D using neon, argon and krypton seeding. These experiments focused on the role of impurities in reducing the $\mathrm{H}$ mode edge pedestal pressure and pressure gradients [17]. They are characterized by significantly lower mantle radiation than those in Fig. 1 and will not be discussed in this article.

In Sections 2 and 3 we will discuss the characteristics of the two types of impurity seeded discharge shown in Fig. 1, focusing on specific discharges which are typical for each type. Various aspects of these discharge types are discussed, i.e. radiation, global confinement, transport, scaling and impurities, although, due to diagnostic limitations, not all topics are covered in all sections. A discussion of the experimental results, comparison with other machines and conclusions are contained in Section 4.

\section{Impurity seeding in puff and pump ELMing $H$ mode discharges}

Impurity seeding has been applied to ELMing $\mathrm{H}$ mode discharges with a high deuterium flow, resulting in no loss of $\mathrm{H}$ mode confinement and in some cases a small increase, see, for example, Fig. 1. After impurity injection, density increases and a spontaneous transition, described later, is sometimes 


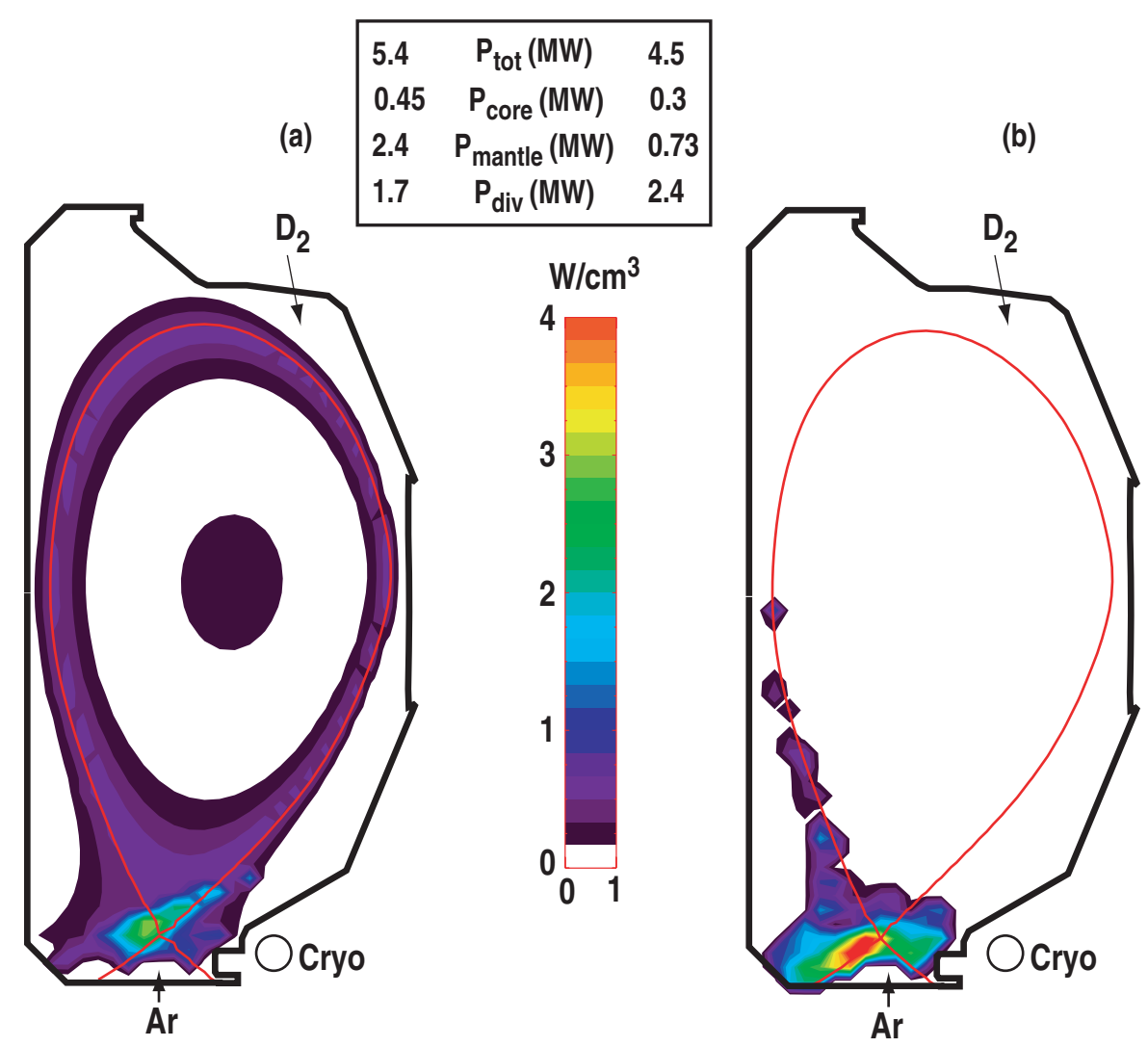

Figure 2. Radiated power profiles at $t=3.24 \mathrm{~s}$ for two puff and pump discharges: (a) radiating mantle, No. 95011; (b) radiating divertor, No. 95012. The discharge para-meters are $I_{p}=1.3 \mathrm{MA}, B_{T}=2.1 \mathrm{~T}, \bar{P}_{N B}=6.3 \mathrm{MW}$ (a) and $6.7 \mathrm{MW}$ (b). The argon and deuterium gas puffing rates were identical for these two discharges.

observed. In the following discussion we will describe changes in the mantle, core and $\mathrm{H}$ mode transport barrier regions with impurity seeding.

Both radiating mantle and radiating divertor discharges have been observed under conditions of high deuterium gas flow and impurity injection. These impurity entrainment experiments used non-intrinsic impurities as a means to obtain a high radiative fraction without a large increase in core impurities. They were accomplished by utilizing a large deuterium gas flow such that the frictional force associated with this flow was larger than the thermal gradient force. The divertor strike point was positioned near the entrance to the toroidally continuous liquid helium cryopump for particle removal. This technique is referred to as puff and pump [16], and the geometry is shown in Fig. 2. While the original goal of these experiments was to produce a highly radiating divertor (e.g., Fig. 2(b)), some discharges instead exhibited a radiating mantle (e.g., Fig. 2(a)). In this radiating divertor discharge, $2.4 \mathrm{MW}$ is radiated in the divertor region and only 1.0 MW is radiated inside the LCFS. A similar discharge exhibits different behaviour; the peak radiated power is reduced and the largest fraction of radiated power, $2.85 \mathrm{MW}$, is now inside the LCFS. Differences between radiating mantle and radiating divertor discharges will be discussed in more detail in Section 2.6.

\subsection{Temporal evolution of ELMing H mode discharges with argon injection}

A discharge with impurity seeding is compared to a puff and pump reference discharge in Fig. 3. Both had a high deuterium gas flow (Fig. 3(f)) and during the interval shown in Fig. 3 were in ELMing $\mathrm{H}$ mode. The discharge with argon injection exhibits higher normalized confinement (solid line) and a monotonic density increase, reaching $\bar{n}_{e} / n_{G W} \approx 0.9$ before the onset of $n=2$ MHD activity. Although density can approach the Greenwald density limit in these puff and pump discharges, stored magnetic energy, $W_{M H D}$, and energy confinement time are the same as, or higher than, in discharges with no impurity seeding, for example, Fig. 3(b). Note that even after the $n=2$ MHD activity begins (Fig. 3(e)), the density is still approximately $30 \%$ above that of the reference case and ELM-free $\mathrm{H}$ mode confinement, $H_{93 P} \geqslant 1$, is maintained. In discharge 96981 there is a transition from 2.95 to $3.1 \mathrm{~s}$ where both density and $\gamma_{\text {mantle }}$ increase at their highest rates. Although density and mantle radiation both increase, there is little or no change in global confinement (Fig. 3(b)). We will refer to this as the spontaneous transition in this article and it will be discussed in more detail later.

After the spontaneous transition, $t>3.1 \mathrm{~s}, \gamma_{\text {mantle }}$ actually decreases while $Z_{\text {eff }}$ begins increasing in Fig. 3 . This is because line radiation is the largest contributor to radiated power and is from low ionized charge states of argon which occur in the plasma edge, $\rho>0.75$, while the densities of $\mathrm{Ar}^{16+}, \mathrm{Ar}^{17+}$ and $\mathrm{Ar}^{18+}$ are the largest contributors to $Z_{e f f}$ and 


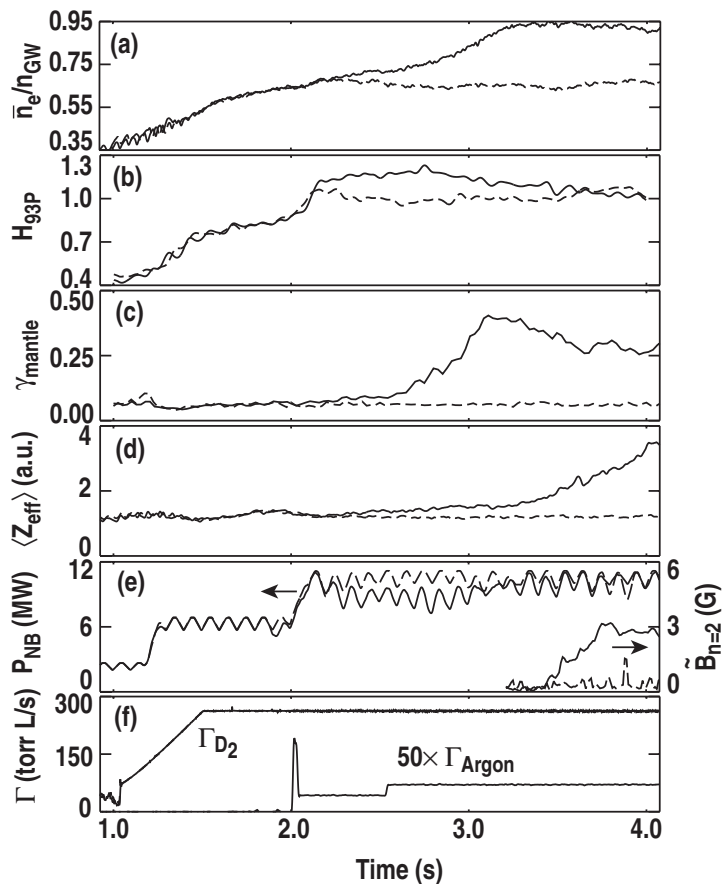

Figure 3. Comparison of an argon impurity seeded discharge (solid curves, No. 96981) with a baseline discharge with no impurity injection (dashed curves, No. 96982). Normalized density and energy confinement are plotted in (a) and (b) respectively, the fraction of mantle radiation is shown in (c), average $Z_{\text {eff }}$ in (d) and neutral beam auxiliary heating power in (e). MHD activity (e) limits the maximum achievable density in discharge 96981. Discharges 96981 and 96982 had the same rate of deuterium puffing, (f). The discharge parameters were: LSN divertor, $I_{p}=1.3 \mathrm{MA}$, $B_{T}=2.1 \mathrm{~T}$.
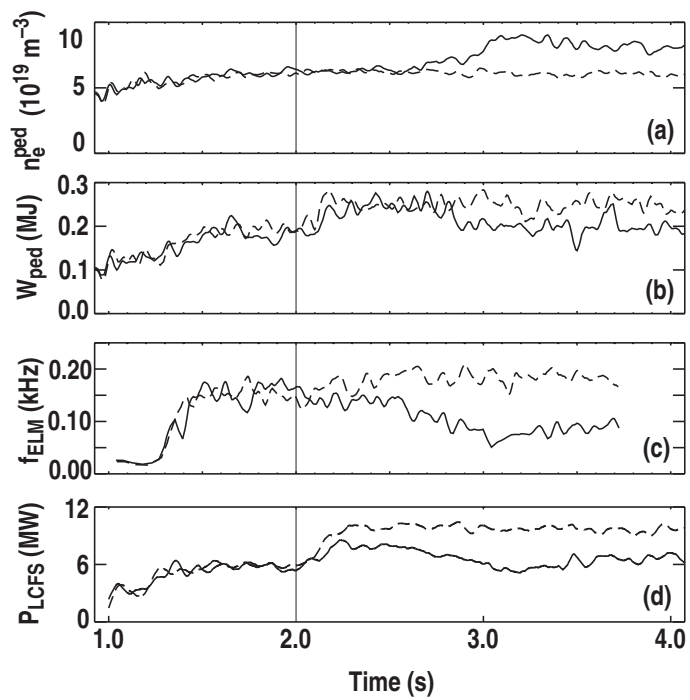

Figure 4. Edge parameters for a radiating mantle discharge (solid curve, No. 96981) and a baseline discharge (dashed curve,

No. 96982). Shown are: (a) pedestal density, (b) pedestal stored energy defined by Eq. (2), (c) ELM frequency, (d) power flow across the LCFS. The discharge parameters are given in the caption to Fig. 3. Argon injection, indicated by a vertical line, begins at $t=2.0 \mathrm{~s}$

have a much smaller fraction of line radiation. Continuum radiation (bremsstrahlung) is not a significant radiator in these

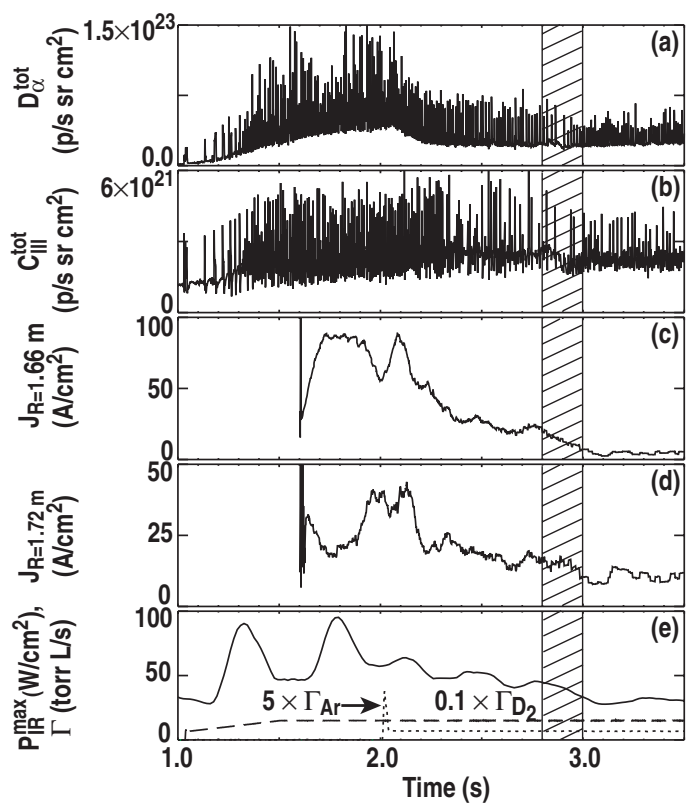

Figure 5. SOL and recycling parameters for a puff and pump radiating mantle discharge (No. 95011). The discharge parameters are given in the caption to Fig. 2. (a) Total deuterium recycling and (b) total C III line emission are plotted before and during argon injection. The incident particle fluxes at the divertor floor are shown: (c) within $1 \mathrm{~cm}$ of the outer strike point, (d) in the SOL near the entrance to the cryopump. The peak heat flux on the divertor tiles measured by an IRTV camera is plotted in (e).

types of discharge. Although the impurity density profile of argon is not measured, by assuming that this profile has the same shape and relative temporal changes as the electron density profile, the decrease in $\gamma_{\text {mantle }}$ is consistent with the changes in the argon density profile. MIST modelling of a similar discharge is presented in Section 2.4.

Some edge characteristics of the two discharges in Fig. 3 are displayed in Fig. 4. The edge electron pedestal density, $n_{e}^{\text {ped }}$ (Fig. 4(a)), is calculated using a hyperbolic tangent fit function [18] to the Thomson scattering profile data. Using the formulation given by Fishpool [19], the pedestal stored energy $W_{\text {ped }}$, shown in Fig. 4(b), is defined by the relation

$$
W_{\text {ped }}=2 P_{e}^{\text {ped }} V_{p}
$$

where $V_{p}$ is the plasma volume in $\mathrm{m}^{3}$ and $P_{i}^{\text {ped }}=P_{e}^{\text {ped }}$ is assumed.

After impurity injection there is only a small decrease in $W_{p e d}$ compared with the reference discharge $\left(W_{\text {ped }} / W_{M H D} \approx\right.$ 0.2 ). Note that although electron pedestal density is initially unchanged after impurity injection there is a marked increase from 2.95 to $3.1 \mathrm{~s}$ associated with the spontaneous transition.

The power flow across the last closed flux surface, $P_{L C F S}$, initially increases because $P_{N B}$ is increased (Fig. 3(e)) near the time of impurity injection. After this initial increase, both the ELM frequency and $P_{L C F S}$ decrease, plotted in Figs 4(c) and (d), where

$$
\begin{aligned}
& P_{L C F S}=P_{N B}+P_{\text {ohmic }}-d W_{M H D} / d t \\
& -P_{\text {mantle }}-P_{\text {core }} .
\end{aligned}
$$

Although there is a significant reduction in this power flow, the argon seeded discharge in Figs 3 and 4 is not completely 


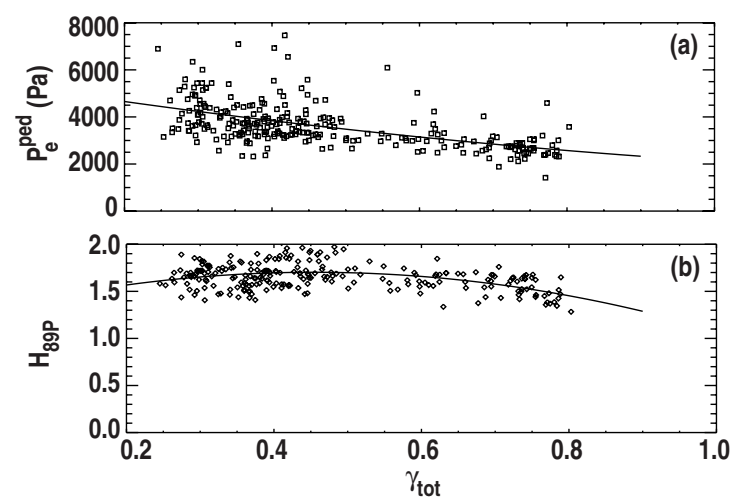

Figure 6. (a) Edge electron pedestal pressure and (b) normalized confinement for puff and pump discharges during the time of argon injection shown as functions of normalized radiated power. The data are from discharge series Nos 95007-95026 $\left(I_{p}=1.3 \mathrm{MA}\right.$, $\left.B_{T}=2.1 \mathrm{~T}, P_{N B}=6-8 \mathrm{MW}\right)$.

detached. Shown in Fig. 5 is the particle flux measured by the floor Langmuir probes near the position of peak heat flux (Fig. 5(c)) and in the outer scrape-off layer (Fig. 5(d)). The particle fluxes are averaged over $0.02 \mathrm{~s}$, so the transient ELM increases are not apparent. Both probes indicate a reduction in the particle flux after impurity injection. Reduced particle flux to the divertor tiles is consistent with a reduction in the peak power flow to these tiles, shown in Fig. 5(e). In Fig. 5(e), the two broad peaks at 1.33 and $1.8 \mathrm{~s}$ are due to ELMs which were not excluded by the automatic ELM filter during processing of the infrared TV (IRTV) data. There is also a decrease in the total $\mathrm{D}_{\alpha}$ line radiation in both the average value and the peak ELM amplitude, shown in Fig. 5(a). The total carbon III line emission, with the same filterscope viewing geometry, does not exhibit the same reduction after argon injection, although some reduction in peak amplitude is observed after the spontaneous transition (Fig. 5(b)). The decrease in the observed $\mathrm{D}_{\alpha}$ line intensity may be due to increasing particle confinement which reduces the incident flux of deuterons to the wall and hence the number of recycled deuterons. The physical mechanisms producing carbon in DIII-D are not well understood, and no conclusions can be inferred about the wall carbon sources at this time.

\subsection{Global scaling characteristics of puff and pump discharges with argon injection}

Non-intrinsic impurities can also affect edge pressure gradients. Figure 6(a) shows the electron pedestal pressure, determined from a hyperbolic tangent fit to the edge electron profiles obtained from Thomson scattering [18]. This series of puff and pump discharges had similar parameters $\left(I_{p}, B_{T}\right.$ and $\left.P_{N B}\right)$ and was obtained during one experimental day. Data near an ELM transition were excluded. There is a systematic decrease in the edge pedestal pressure as the radiated power fraction increases. There is only a small decrease in $H_{89 P}$ at the highest radiated power, plotted in Fig. 6(b). The decrease in edge pressure suggests that impurity injection may be a useful tool for pressure profile control in future scenarios such as that of the DIII-D advanced tokamak programme [20]. Modelling of similar discharges shows a reduction of edge bootstrap

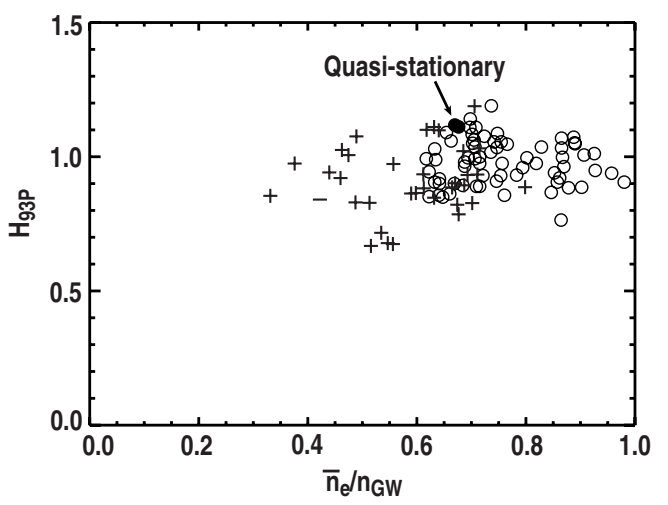

Figure 7. Puff and pump argon seeded discharges (circles) have been achieved at higher densities than those for comparable non-seeded DIII-D ELMing H mode discharges (crosses) taken from the ITER ELMing database, $1.2 \leqslant I_{p} \leqslant 1.4$ MA. Two times during a steady state puff and pump argon seeded discharge are also displayed (solid circles).

current with impurity puffing, and the total edge toroidal current also decreased with increasing impurity injection.

DIII-D puff and pump ELMing H mode discharges allow operation at higher densities than unseeded discharges, shown in Fig. 7. The non-impurity discharges are taken from the DIIID ITER ELMing H mode database. The physical mechanisms limiting the maximum density in these non-seeded discharges are not well understood. The maximum density appears to be determined by the $\mathrm{H}$ mode boundary plasma and is limited by either a collapse of the $\mathrm{H}$ mode edge pedestal or divertor detachment. In general, the argon discharges allow operation with confinement enhancements equivalent to ELMing H mode but at higher densities than normally obtained in DIII-D and is due, at least in part, to larger density peaking. We note that to date the highest density discharges with argon injection achieved in DIII-D are not steady state. However, quasistationary impurity seeded discharges have been obtained. One example is shown in Fig. 7 as solid circles, taken at two times (2.8 and $3.4 \mathrm{~s}$ ). Many discharges, while not stationary, maintain good confinement as the density slowly increases over several confinement times. An example of this behaviour is the puff and pump discharge shown in Fig. 1, where $0.75 \leqslant$ $\bar{n}_{e} / n_{G W} \leqslant 0.87$ and $H_{93 P} \geqslant 1.1$ for a time $\Delta t / \tau_{E}=5.5$. The data with impurity seeding plotted in Fig. 7 show no trend of increasing confinement with density, and at a given density there are variations in $H_{93 P}$ from discharge to discharge of more than $\pm 10 \%$. The points displayed in this figure represent one or two times during the impurity seeded phase.

\subsection{Transport}

Puff and pump discharges show a marked decrease in the effective thermal diffusivity with impurity injection with the largest change occurring in the mantle region. Reductions in other transport channels, for example, momentum, are also observed, accompanied by an increase in the thermal neutron rate. The transport coefficients were calculated using the TRANSP code [21]. The inputs to this code are: $T_{e}$ (Thomson scattering and third harmonic electron cyclotron Fourier spectrometer (HECE)), $n_{e}$ (Thomson scattering and four channel interferometer), $T_{i}$ (charge exchange recombination 

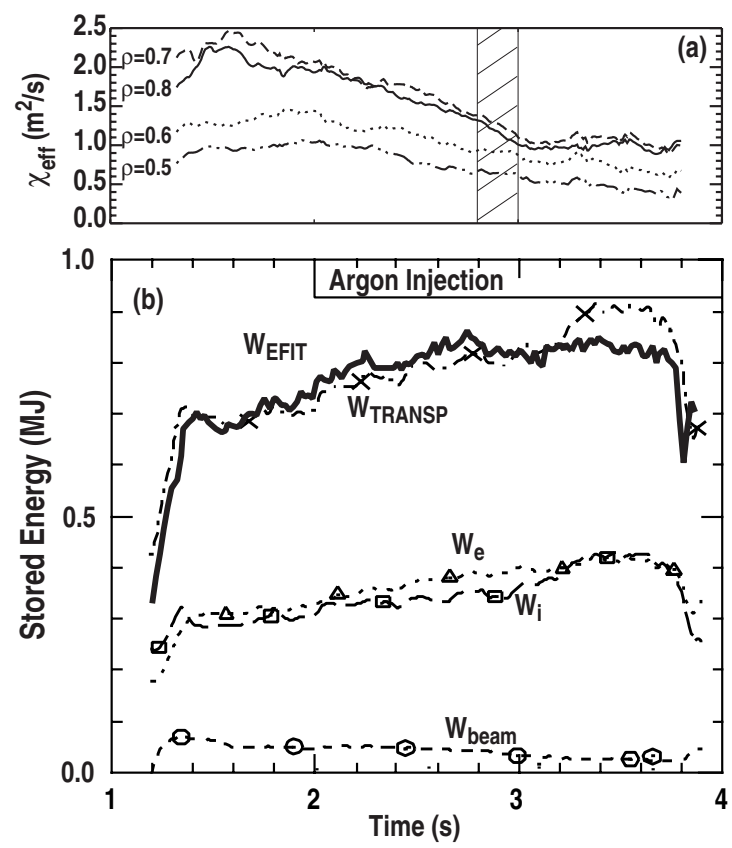

Figure 8. (a) Temporal behaviour of $\chi_{\text {eff }}$ at four radii in the mantle region (discharge 95011). (b) Total stored energy $W_{E F I T}$, calculated from the EFIT magnetic equilibrium code (solid line), is compared with $W_{\text {TRANSP }}$. The components $W_{e}, W_{i}$ and $W_{\text {beam }}$ are also plotted.

(CER)), radiated power (48 channel bolometer array), impurity density (SPRED UV spectrometer and CER), magnetic equilibria (magnetic probes and flux loops), current profile (35 channel motional Stark effect (MSE) array) and neutron emission $S_{n}$.

The single fluid effective thermal diffusivity $\chi_{\text {eff }}$ is shown in Fig. 8(a) for a typical high density puff and pump discharge. Before argon injection $\chi_{\text {eff }}$ decreases in the outer mantle region ( $\rho=0.7$ and 0.8$)$ and is nearly constant or increases slightly further inside $(\rho=0.5$ and 0.6$)$. However, after argon injection, $\chi_{e f f}$ decreases over the entire mantle region. Values of $\chi_{\text {eff }}$ for $\rho \geqslant 0.9$ are not plotted because of large uncertainties in the TRANSP calculation due to uncertainties in the radiation and neutral particle profiles in this region. The short electron-ion equilibration time in these high density discharges allows near equilibration between ions and electrons and thus $T_{i} \approx T_{e}$. Since the calculation of $\chi_{e f f}$ has lower uncertainties than $\chi_{i}$ and $\chi_{e}$, and $\chi_{e f f}$ can be uniquely determined, it is presented in Fig. 8(a). However, both $\chi_{i}$ and $\chi_{e}$ decrease after argon injection.

The total stored energy exhibits a modest increase after impurity injection begins at $2.0 \mathrm{~s}$, shown in Fig. 8(b). $W_{E F I T}$, calculated by the EFIT magnetic equilibrium code, is compared with the stored energy $W_{T R A N S P}$, calculated from the TRANSP profile analysis, and is in good agreement. Also shown in Fig. 8(b) are the stored energy components contributing to the total stored energy from electrons, thermal ions and fast ions. We note that the thermal component $W_{i}+W_{e}$ is $\geqslant 91 \%$ of the total during this discharge, i.e. the fast ion contribution is small. Thermal pressure profiles (not shown) indicate that the core region exhibits the largest increase in both ion and electron pressure leading to the increase in stored energy described previously. After the spontaneous transition

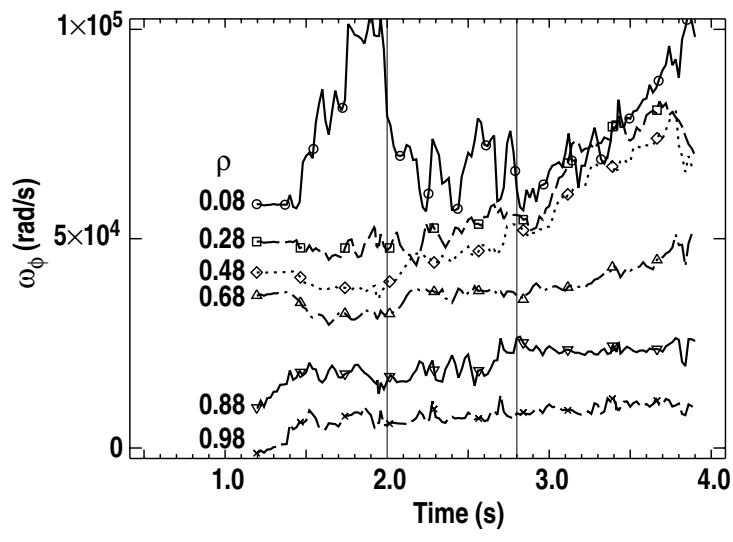

Figure 9. Toroidal plasma rotation at six radii as a function of time (discharge 95011). The vertical lines show the beginning of impurity injection at $2.0 \mathrm{~s}$ and the spontaneous transition beginning at $2.8 \mathrm{~s}$.

beginning at $t \approx 2.8 \mathrm{~s}$, the central ion pressure further increases while central electron pressure remains relatively constant.

Toroidal rotation increases after impurity injection and this increase is most apparent after the spontaneous transition (Fig. 9). After this time, the region of highest rotational shear is in the mantle region, while the highest rotation is observed in the core. Since core ion and electron density are also higher we conclude that core momentum confinement is increasing. In addition to the increases in core toroidal rotation after impurity injection, there is also a large increase in core rotation, most apparent at $\rho=0.08$ in Fig. 9, beginning at $1.45 \mathrm{~s}$, peaking at $1.78 \mathrm{~s}$ and then rapidly collapsing. This phenomenon occurs before impurity injection and is observed even in some discharges without impurity puffing. The cause of this transient behaviour has not yet been identified but it could be associated with the formation of an internal transport barrier (ITB) in the plasma core. This phenomenon terminates before impurity injection and will not be discussed further.

An increase in toroidal rotational shear can contribute to $\boldsymbol{E} \times \boldsymbol{B}$ shear stabilization of microturbulence and hence lower diffusivities [22]. However, for these puff and pump discharges, measurements of density fluctuations associated with this microturbulence were not possible due to the interference of ELMs and the lower initial turbulence levels in $\mathrm{H}$ mode. Hence no conclusions about the relation between toroidal rotation, $\boldsymbol{E} \times \boldsymbol{B}$ shear stabilization and lower diffusivity can be made. We note, however, that an increase in core toroidal rotation always accompanies the spontaneous transition in this type of discharge.

\subsection{Impurities in puff and pump ELMing $H$ mode discharges}

The intentional introduction of impurities has the potential for increasing both $Z_{e f f}$ and dilution of the main species to unacceptable levels, lowering reactivity and neutron yield. Thus, core impurity concentration is an important consideration in these types of discharge.

Shown in Fig. 10 is $Z_{\text {eff }}$, calculated by the TRANSP code with measured impurity density profiles, for puff and pump discharge 95011. Although $Z_{e f f}$ increases after argon injection at $2.0 \mathrm{~s}$, the profile is hollow and $Z_{\text {eff }}$ actually decreases after the spontaneous transition at $2.8 \mathrm{~s}$. This 


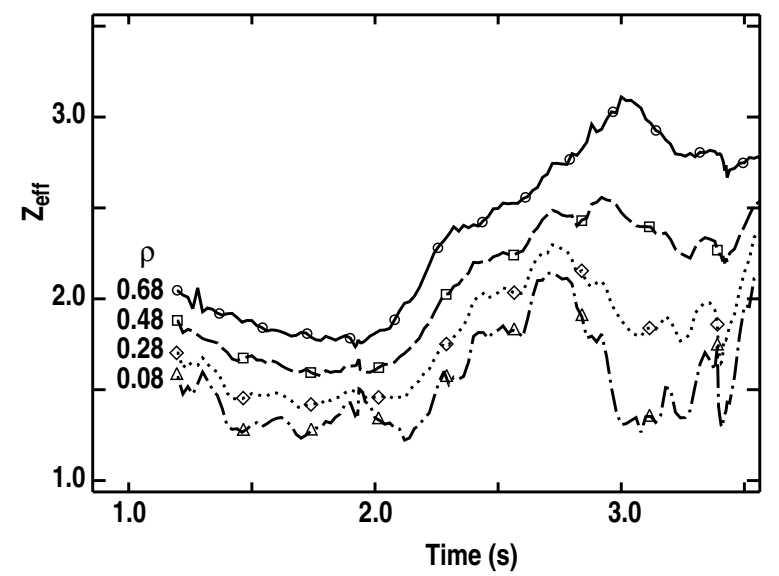

Figure 10. Evolution of $Z_{e f f}$ calculated from the TRANSP code (for discharge 95011). Symbols are used to identify the different curves and do not represent discrete time calculations.
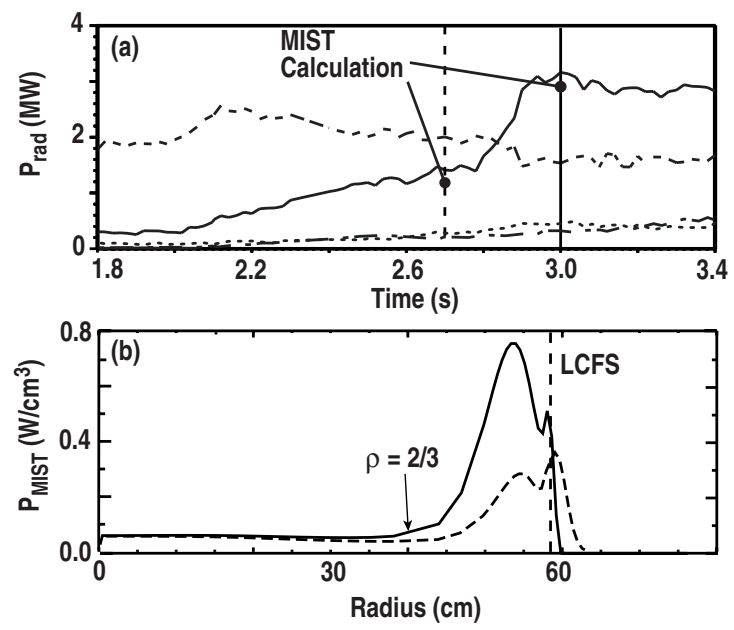

Figure 11. (a) Radiated power in various regions: $\rho<1 / 3$ (dotted curve), $1 / 3<\rho \leqslant 2 / 3$ (chain curve), $2 / 3<\rho<1$ (solid curve) and divertor (dashed curve) for discharge 95011 . MIST calculations of radiated power integrated from $\rho=2 / 3$ to $\rho=1$ at two times (solid and dashed vertical lines), before and after the spontaneous transition, are also shown. (b) Radiated power profiles calculated from the MIST code for two times: $2.7 \mathrm{~s}$, dashed curve; $3.0 \mathrm{~s}$, solid curve.

decrease in $Z_{\text {eff }}$ is consistent with an increase in the thermal neutron rate, calculated by the TRANSP code.

The MIST 1-D impurity transport code [23] has been used to calculate radiated power from all charge states of argon, carbon and oxygen using measured electron density and temperature profiles and SPRED UV impurity line intensities. Agreement with bolometric measurements, shown in Fig. 11(a), is very good. Radiation profiles for the two times before and after the spontaneous transition are shown in Fig. 11(b). Even after impurity injection, radiation is confined to the mantle region.

\subsection{MHD activity in puff and pump discharges}

Nearly all puff and pump impurity seeded discharges exhibit $n=2$ MHD activity which limits the highest density and magnetic stored energy. Analysis of the MHD in

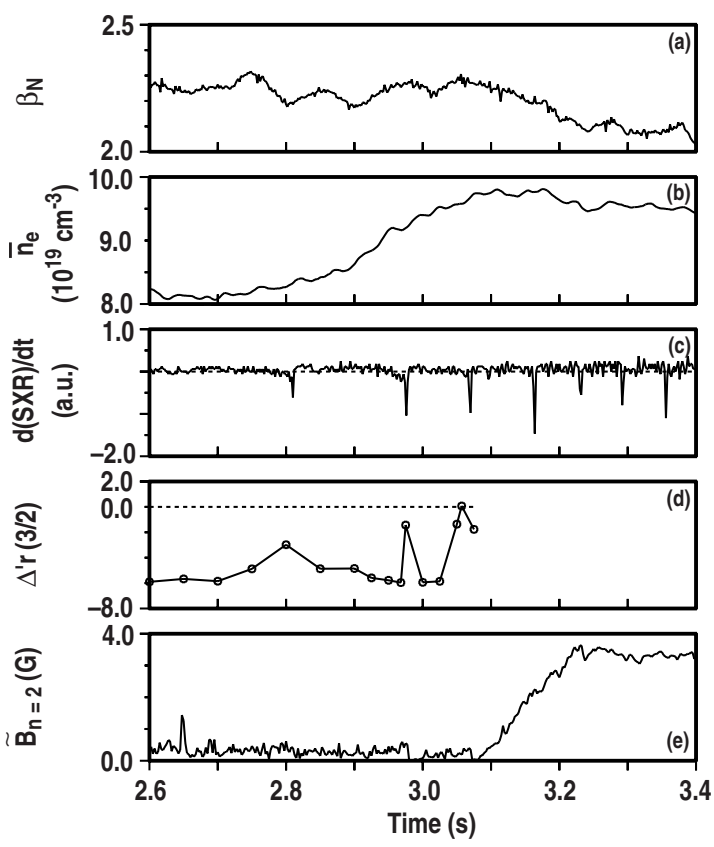

Figure 12. (a) Normalized $\beta$ and (b) electron density decrease after the onset of $n=2$ MHD activity. Before the first three sawteeth, shown by (c) (the time derivative of a central soft $\mathrm{X}$ ray channel), $\Delta^{\prime} r$ becomes successively more positive, i.e. unstable until (e) MHD activity commences. $\Delta^{\prime} r(\mathrm{~d})$ is calculated from the TEAR code.

a discharge with the same characteristics as No. 95011 (discussed previously) is displayed in Fig. 12. This discharge was selected because the fast time domain magnetic data were available during the onset of the mode. Modal analysis identifies the activity as $m=3, n=2$ and its onset occurs just after $\Delta^{\prime} r$ becomes less negative, i.e. more unstable (Fig. 12(d)). $\quad \Delta^{\prime} r$ is calculated from the code TEAR [24] using EFIT equilibria as input. In the case shown in Fig. 12, $\Delta^{\prime} r$ increases in amplitude before each sawtooth crash, shown as a negative spike in Fig. 12(c), until $t=3.06 \mathrm{~s}$ when the $3 / 2$ tearing mode becomes unstable. This mode is identified as a neoclassical tearing mode where the helically perturbed bootstrap current from the $q=1$ sawtooth coupling to the $q=3 / 2$ mode excites the unstable mode. The resulting sawtooth reconnection can provide the necessary seed island and, in fact, the $3 / 2$ mode is observed to begin increasing in amplitude within $0.02 \mathrm{~s}$ after a sawtooth crash. Although sawteeth are not always the trigger for the increase in $3 / 2 \mathrm{MHD}$ activity, $n=2$ modes are observed in all types of impurity seeded discharge and usually limit the maximum density and confinement achieved.

\subsection{Differences between radiating mantle and radiating divertor discharges}

The effects of impurity injection into puff and pump discharges occur in two categories: (a) gradual changes observed immediately after argon injection and (b) more pronounced changes occurring at the spontaneous transition. Both of these are discussed in this section.

Immediately after impurity injection, mantle radiation begins increasing (Fig. 3) and decreases in ELM frequency, peak divertor heat flux and divertor particle fluxes are observed 

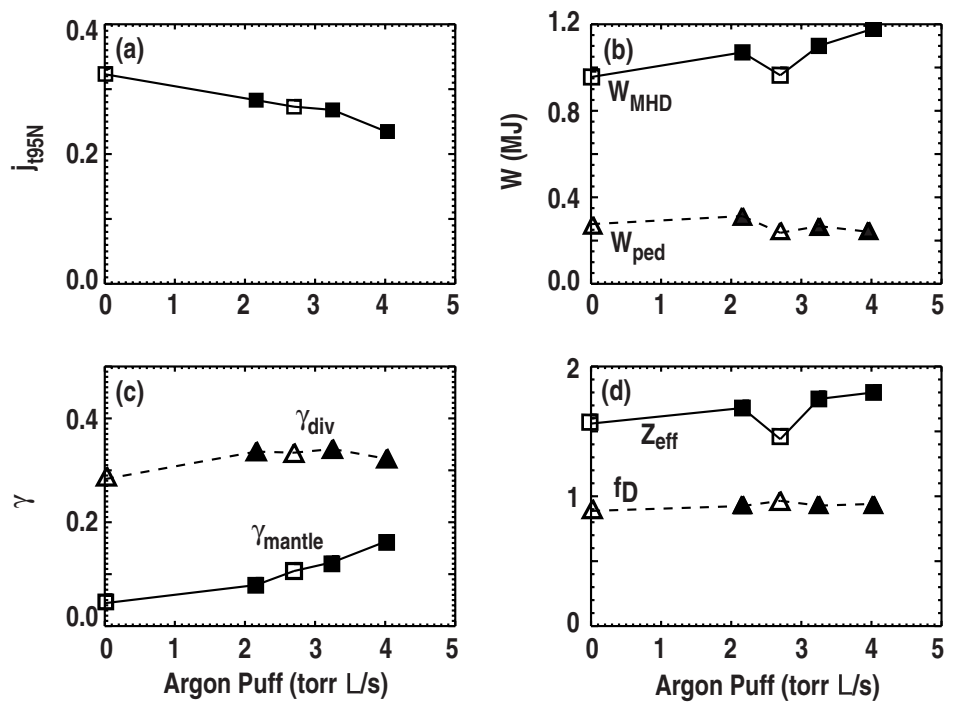

Figure 13. Impurity puff scan for five discharges with similar characteristics: $I_{p}=1.3 \mathrm{MA}, B_{T}=2.1 \mathrm{~T}, P_{N B}=12 \mathrm{MW}$. The parameters are calculated at $t=2.6 \mathrm{~s}$ : (a) normalized edge current density from EFIT reconstructions with MSE, (b) $W_{M H D}$ and $W_{p e d}$, (c) divertor and mantle radiated power fractions, (d) core $Z_{e f f}$ and core deuteron fraction $f_{D}$. Discharges with $n=2$ MHD activity during or immediately before $t=2.6 \mathrm{~s}$ are shown as open symbols. A constant argon gas injection rate commenced at $2.0 \mathrm{~s}$.

(Figs 4 and 5). There is also usually a modest increase in confinement and density.

In this phase, higher argon puff rates can increase mantle radiation and stored energy, while reducing the edge current density, shown in Fig. 13. The normalized edge toroidal current density at $\rho=0.95, j_{t} 95 \mathrm{~N}$, monotonically decreases with increasing argon (Fig. 13(a)) and is unaffected by the presence of $n=2$ MHD activity, shown as open symbols. This current density is determined with the EFIT magnetic equilibrium code including MSE measurements. Internal inductance $l_{i}$ shows little change (not plotted) as the puffing rate is varied. There is also an increase in stored energy with impurity injection, shown in Fig. 13(b), except for the discharge where an $n=2$ mode is present. However, the pedestal energy $W_{p e d}$ is nearly constant. For this scan there is only a small increase in the central $Z_{\text {eff }}$ (Fig. 13(d)) as the argon flow rate is increased and only a small decrease in the fraction of core deuterons $f_{D}$ inferred from the SPRED UV spectrometer and electron density profiles, where $f_{D}$ is defined as $f_{D}=1-\Sigma n_{i} Z_{i} / n_{e}$. Since these discharges are evolving, the data in Fig. 13 are evaluated at a fixed time, $2.6 \mathrm{~s}$ in all discharges.

The second category defining impurity seeded discharges occurs during and after the spontaneous transition. This is a rather broad transition occurring over approximately 150 $200 \mathrm{~ms}$. During this transition, the rate of density rise increases and the fraction of mantle radiation increases rapidly, typically $\gamma_{\text {mantle }}>0.3$ at the end of the spontaneous transition (Figs 2 and 3). There is a marked decrease in $\chi_{\text {eff }}$ (Fig. 8) and core toroidal rotation begins increasing (Fig. 9) while core $Z_{\text {eff }}$ decreases (Fig. 10).

A spontaneous transition is not observed in all discharges; some continue with a radiating divertor. However, once conditions for the spontaneous transition have been established, radiating mantle discharges are quite reproducible. The conditions required are a high deuterium flow injected above the midplane (see the geometry in Fig. 2), typically
150 torr L/s or greater, and a sufficiently high flow of argon, injected into the divertor region. The argon flow rate to obtain this spontaneous transition depends upon the neutral beam heating power, but to date there are insufficient data to establish a threshold scaling relation for argon injection as a function of heating power.

\section{Impurity seeding in $\mathrm{L}$ mode discharges}

In Section 2 we described puff and pump ELMing $\mathrm{H}$ mode discharges with argon injection. L mode discharges, both diverted and inner wall limited, have also been obtained in DIII-D with neon seeding. A goal of this work is to extend the results of TEXTOR radiative improved (RI) mode to larger devices such as DIII-D and also to different configurations such as a divertor. In this section we will discuss L mode discharges with enhanced confinement comparable to $\mathrm{H}$ mode. Such discharges have been obtained in both limiter and divertor configurations, at densities lower than the puff and pump discharges in Section 2. First, IWL L mode discharges are presented and then USN L mode diverted discharges, with more complete diagnostic coverage, are analysed.

\subsection{IWL L mode discharges with impurity seeding}

The TEXTOR RI mode has an L mode edge, a nearly circular cross-section and is limited by the ALT-II pumped limiter on the outboard radius [25]. Similar shapes have been achieved in DIII-D, with the exception that DIII-D discharges are limited on the inner graphite tiled centre post and there is no effective pumping in this configuration. Some DIII-D discharges do exhibit behaviour qualitatively similar to the TEXTOR RI mode (see, e.g., Fig. 14). In this discharge, neutral beam heating is applied at about the time of current flat-top, $1 \mathrm{~s}$. The confinement enhancement $H_{89 P}$ (not shown) is $\approx 1.2$, or nearly L mode, until shortly after neon injection at $1.2 \mathrm{~s}$. At this point, the magnetic stored energy $W_{M H D}$, density 


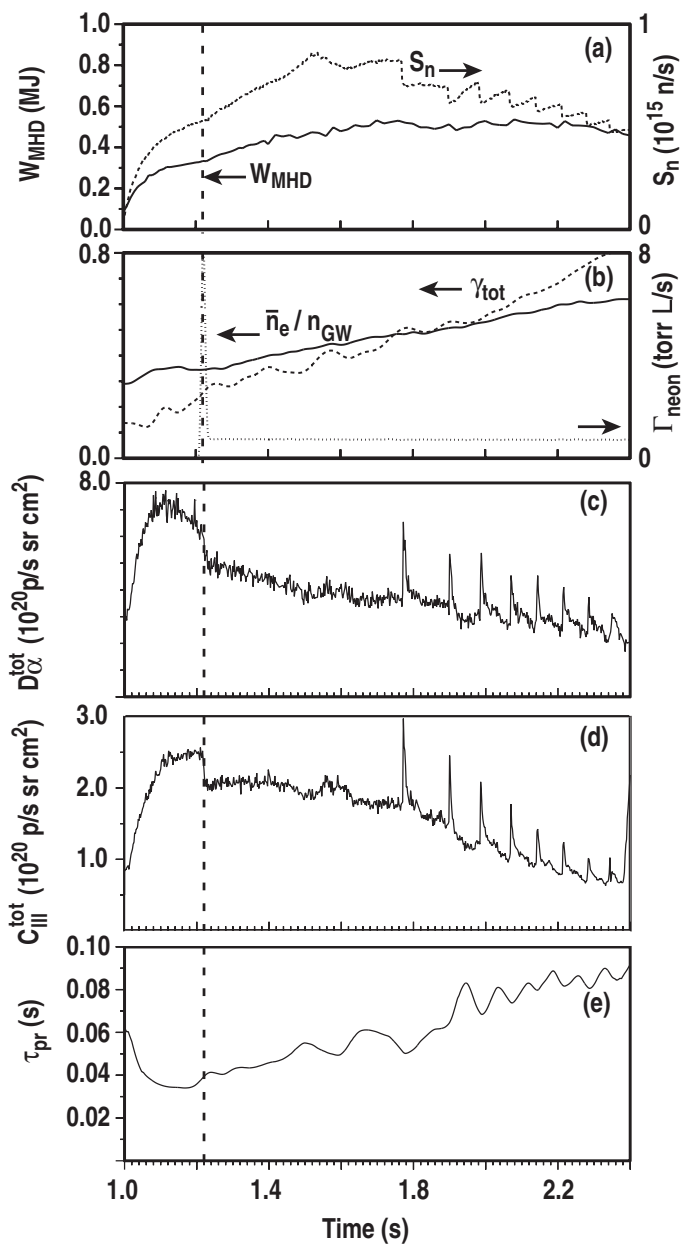

Figure 14. (a) Stored magnetic energy increases for an IWL L mode discharge (No. 98347) after (b) neon injection begins at $1.22 \mathrm{~s}$. The neutron rate $S_{n}$, (a), initially increases and then remains constant until the onset of sawteeth. Both (b) mantle radiation and density increase while (c) the $\mathrm{D}_{\alpha}$ and (d) the C III line intensities are steadily reduced. The spikes in $\mathrm{D}_{\alpha}$ and $\mathrm{C}$ III correspond to sawtooth reconnection events. The relative particle confinement time (Eq. (5)) is plotted in (e). The parameters are: inner wall limited, $I_{p}=1.0 \mathrm{MA}, B_{T}=2.1 \mathrm{~T}, P_{N B}=4.9 \mathrm{MW}, \kappa=1.24-1.27$

and radiated power (Figs 14(a) and (b)) increase until the onset of sawtooth activity at $1.75 \mathrm{~s}$. After this time, density and radiated power continued rising (there was no active pumping of this discharge), but $W_{M H D}$ becomes nearly constant and then slowly decreases. The total $\mathrm{D}_{\alpha}$ and C III line intensities, inferred from a 16 channel filterscope array for an IWL DIII-D discharge, are shown in Figs 14(c) and (d), respectively. After neon injection, there is a prompt drop in $\mathrm{D}_{\alpha}$ and C III intensity and then $\mathrm{D}_{\alpha}$ emission steadily decreases. With the onset of sawteeth at $1.76 \mathrm{~s}$, the C III intensity also decreases.

For DIII-D IWL discharges an absolute calculation of particle confinement time is difficult due to the limited diagnostics observing the inner wall. Nevertheless an estimate can be made from the $\mathrm{D}_{\alpha}$ flux using the Johnson and Hinnov relation $\Gamma_{D R} / \Gamma_{D_{\alpha}^{t o t}} \approx 15$ [26], where $\Gamma_{D R}$ is the recycling flux. The total $\mathrm{D}_{\alpha}$ emission is given by

$$
\mathrm{D}_{\alpha}^{t o t}=\int D_{\alpha} d S
$$
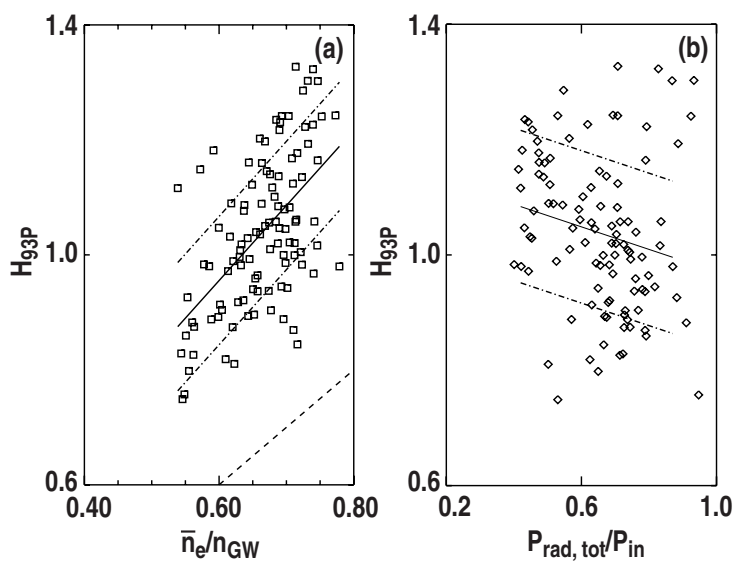

Figure 15. (a) The confinement enhancements $H_{89 P}$ from the DIII-D radiating mantle database for IWL L mode discharges with impurity seeding in DIII-D are above the TEXTOR RI mode scaling relation (dashed line). (b) Confinement plotted as a function of $P_{\text {rad }, \text { tot }} / P_{\text {in }}$. The solid lines are an offset linear fit and the chain lines correspond to one standard deviation $\left(I_{p}>1.0 \mathrm{MA}, \gamma_{\text {tot }}>0.4\right.$ (data being taken during the time of impurity injection)).

and is approximated by the $\mathrm{D}_{\alpha}$ line emission from the 16 channel filterscope array integrated over the DIII-D vessel surface $S$. Using this relation, we can then calculate a relative particle confinement time, defined as

$$
\tau_{p r}=N_{D} /\left(\Gamma_{\text {gas }}+\Gamma_{N B}+\Gamma_{D R}-d N_{D} / d t\right)
$$

where $\Gamma_{\text {gas }}$ and $\Gamma_{N B}$ are the external fuelling rates from gas and neutral beams, and $N_{D}$ is the total number of deuterons, calculated from the electron density, $Z_{e f f}$, and plasma volume.

Plotted in Fig. 14(e) is the relative particle confinement time $\tau_{p r}$. There is a nearly monotonic increase in $\tau_{p r}$ after impurity injection. However, due to the assumptions discussed above, an absolute value cannot be ascribed to this measurement and, in particular, the electron temperature at the inner wall must be included in the calculation before a more quantitative representation of particle confinement time can be made.

3.1.1. Confinement scaling of IWL L mode discharges Of the various shapes which can be achieved in DIII-D, inner wall limiter L mode discharges most closely approximate the TEXTOR shape and edge properties. The confinement scaling $H_{93}$ P as a function of normalized density for this discharge type is plotted in Fig. 15(a). The discharges represented in this figure are taken from the radiating mantle impurity database from 1997 to 1999 . The solid line represents an offset linear fit to the data points and the error bars correspond to one standard deviation $\sigma$. Also plotted is the TEXTOR RI mode scaling, given by Eq. (1). The correlation coefficient $r$ for these data is 0.56 but even with this scatter, and within the $1 \sigma$ error bounds, there is an increase of confinement with density, similar to that for TEXTOR. The confinement enhancement $H_{98} P$ is above the TEXTOR empirical scaling, Eq. (1). We note that the DIII-D discharges were evolving in time, while the TEXTOR scaling was derived using quasi-stationary discharges. The correlation coefficient for the data in Fig. 15(b) is -0.20 and thus, as observed in TEXTOR, there is little or no correlation between 


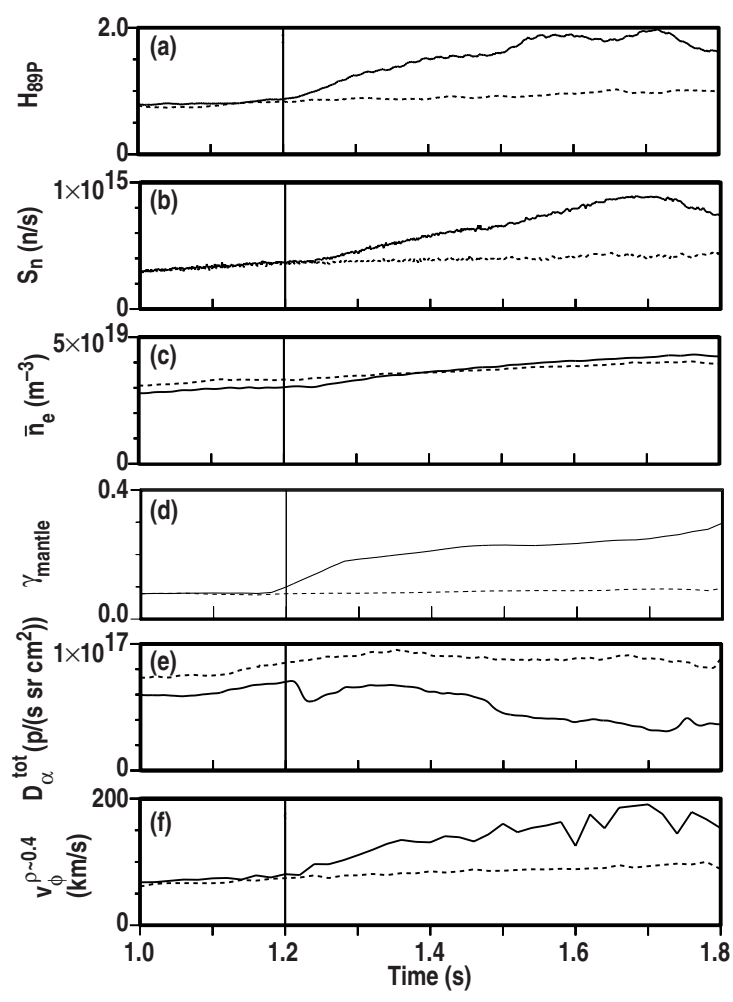

Figure 16. Temporal response for a neon impurity seeded $\mathrm{L}$ mode USN discharge, No. 98782 (solid curves), and a baseline discharge, No. 98783 (dashed curves), with deuterium gas injection to achieve approximately the same density $\bar{n}_{e}$. Plotted are: (a) $H_{89 P}$, (b) neutron emission $S_{n}$, (c) line averaged electron density, (d) $\gamma_{\text {mantle }}$, (e) $\mathrm{D}_{\alpha}$ line intensity, (f) core impurity ion toroidal rotation $\left(I_{p}=1.2 \mathrm{MA}, B_{T}=1.6 \mathrm{~T}, P_{N B}=4.6 \mathrm{MW}\right)$.

radiated power and normalized confinement with impurity seeding. Within the range of radiated powers shown in Fig. 15, $\gamma_{t o t}>0.4$, the DIII-D data do not show a threshold for a transition to improved confinement as observed in TEXTOR where a threshold radiative power fraction, $\gamma_{t o t} \approx 0.5$, has been reported [3].

\subsection{USN diverted L mode discharges with impurity seeding}

Since the mantle region plays such an important role in discharges with impurity seeding, it might be expected that the effects discussed for limiter discharges would also be observed in $\mathrm{L}$ mode diverted discharges. In fact, enhanced confinement L mode discharges have also been obtained in a diverted USN configuration. Such a configuration was chosen because of the higher $\mathrm{L}-\mathrm{H}$ power threshold when the $\boldsymbol{\nabla} \boldsymbol{B} \times \boldsymbol{B}$ drift direction is away from the $\mathrm{X}$ point. (All the discharges discussed in Section 3 are L mode.) Shown in Fig. 16 are a discharge with neon injection and a baseline discharge with deuterium injection, allowing comparison at similar densities (Fig. 16(c)). Beginning immediately after neon injection at $1.2 \mathrm{~s}$, there are marked increases in $H_{89 P}$, neutron rate, radiated power and central toroidal rotation, and a decrease in the total $\mathrm{D}_{\alpha}$ line intensity, plotted in Figs 16(a), (b), (d), (f) and (e), respectively.

3.2.1. USN confinement scaling with impurity seeding and an L mode edge Normalized energy confinement in DIII-D USN

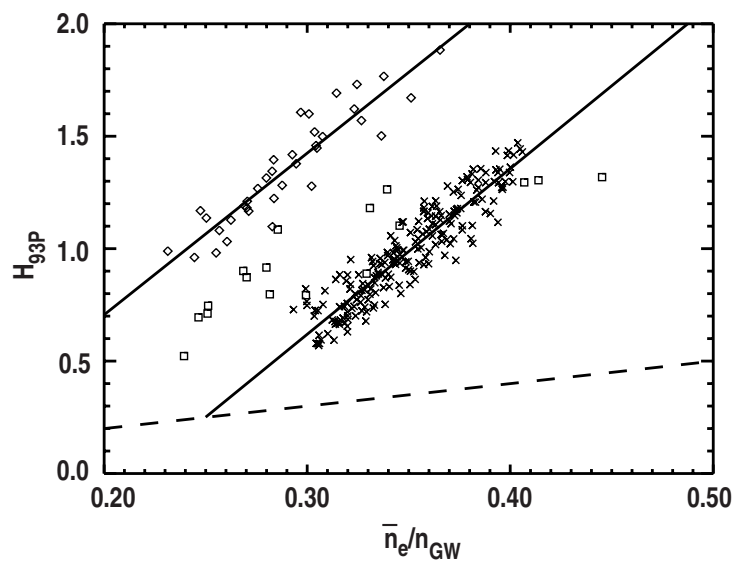

Figure 17. A subset of the experimental points from the USN L mode impurity database for two experimental days: diamonds and squares represent neon impurity seeding from the same day (discharges 96559-96569, $\bar{q}_{95}=4.2$ ) with and without pumping, respectively. The crosses represent experimental points from another day (discharges 99774-99794, $\bar{q}_{95}=3.7$ ). The solid lines are linear offset fits to the diamonds and crosses. TEXTOR RI mode scaling is shown as a dashed line $\left(I_{p}>1.1 \mathrm{MA}, \gamma_{\text {tot }}>0.3\right.$, $P_{N B}>2 \mathrm{MW}$, data taken during the impurity puffing phase).

L mode discharges with impurity seeding is above that given by TEXTOR scaling, but no correlation with either density or radiation has been observed in analysing the entire USN L mode impurity seeded data set. However, there are clusters of points from this data set which do show a correlation. Three sets of data are plotted in Fig. 17 from two experimental days and there are well defined linear correlations between $H_{93} P$ and $\bar{n}_{G W}$ for two cases (diamonds and crosses) with a higher increase in confinement occurring at much lower density than reported in TEXTOR. The differences between these data sets are significant, for example, at $\bar{n}_{e} / \bar{n}_{G W}=0.32, H_{93 P}$ varies by a factor of 2 . The major differences between these data sets are:

(a) Lower gas fuelling, lower recycling and lower target density for the diamond set.

(b) Higher heating power $\left(\left\langle P_{N B}\right\rangle=7.5 \mathrm{MW}\right.$, diamonds; 4.7 MW, crosses).

(c) Outer strike point located closer to the cryo- pump (diamonds), although for both sets the strike point was not positioned for optimal pumping.

We note that higher neutral beam heating power also has higher momentum input, which may allow shear stabilization of microturbulence at lower densities. Shear stabilization is discussed in more detail in Section 3.2.4. Further experiments are required to establish which conditions in the two data sets described above produce the marked differences shown in Fig. 17. The third data set (the squares in Fig. 17) with no pumping does not exhibit as strong a dependence of confinement on normalized density. The discharge parameters and shape were the same as those for the lowest density data set (diamonds).

3.2.2. Transport in USN L mode discharges $\mathrm{L}$ mode discharges with a USN configuration show a marked decrease in thermal diffusivity with impurity injection. These 

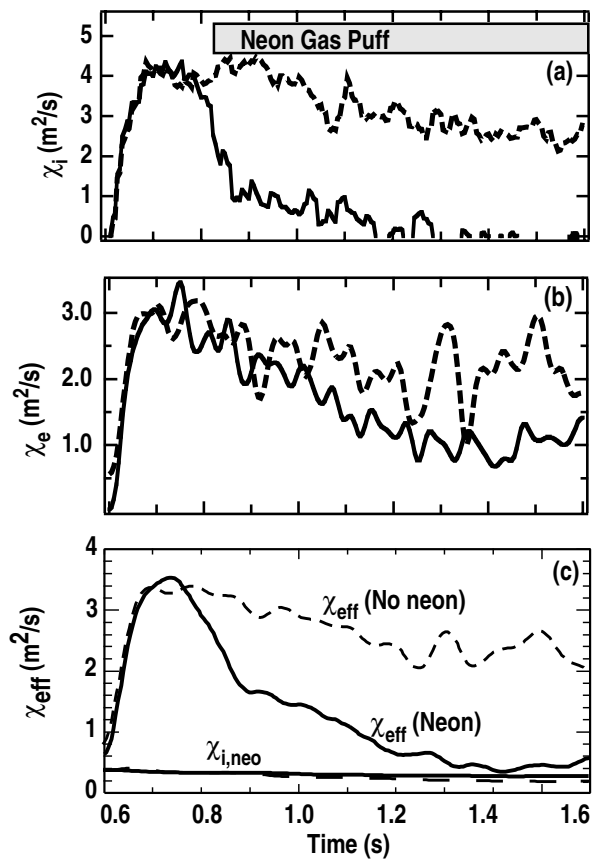

Figure 18. Thermal diffusivities at $\rho=0.65$ as a function of time for an L mode USN neon seeded discharge (solid curves, No. 98775) and a baseline discharge without impurity seeding (dashed curves, No. 98777): (a) $\chi_{i}$, (b) $\chi_{e}$, (c) $\chi_{e f f}$ and $\chi_{i, n e o}$.

discharges have lower densities than those for the puff and pump scenario described in Section 2, allowing calculation of $\chi_{i}$ and $\chi_{e}$ with lower uncertainties. An example for a USN discharge is shown in Fig. 18, and compared with a baseline non-seeded discharge. These discharges are not stationary, for example, $q(0)$ decreases monotonically during the time shown, and hence the diffusivities decrease with time even in the reference case. However, the presence of neon leads to a dramatic change in both $\chi_{i}$ and $\chi_{e f f}$ when compared with the reference discharge, and there is also a modest reduction in $\chi_{e}$. Note that $\chi_{\text {eff }}$ has been smoothed over $150 \mathrm{~ms}$ to better indicate the neoclassical ion diffusivity (Fig. 18(c)). The electron thermal diffusivity is reduced primarily in the mantle region compared with the baseline discharge, while $\chi_{i}$ is lower across almost the whole profile.

3.2.3. Edge particle fluxes in USN L mode discharges Previously an example was shown (Fig. 14) where the particle confinement time increased in an IWL $\mathrm{L}$ mode discharge. Since the $\mathrm{D}_{\alpha}$ behaviour is similar in IWL and USN L mode configurations, increased particle confinement times might be expected in both cases, producing decreases in edge fluctuations and particle fluxes. Although such measurements were not available for the series of IWL discharges in Section 3.1 they have been obtained in L mode USN diverted discharges and are displayed in Fig. 19. Turbulent radial flux and normalized fluctuation levels are reduced by factors of $2-$ 3 in the discharge with impurity seeding. The reduction in particle flux (Fig. 19(b)) is qualitatively consistent with the observed reduction in $\mathrm{D}_{\alpha}$ line emission. The measurements shown in Fig. 19 were made with a fast reciprocating probe [27].

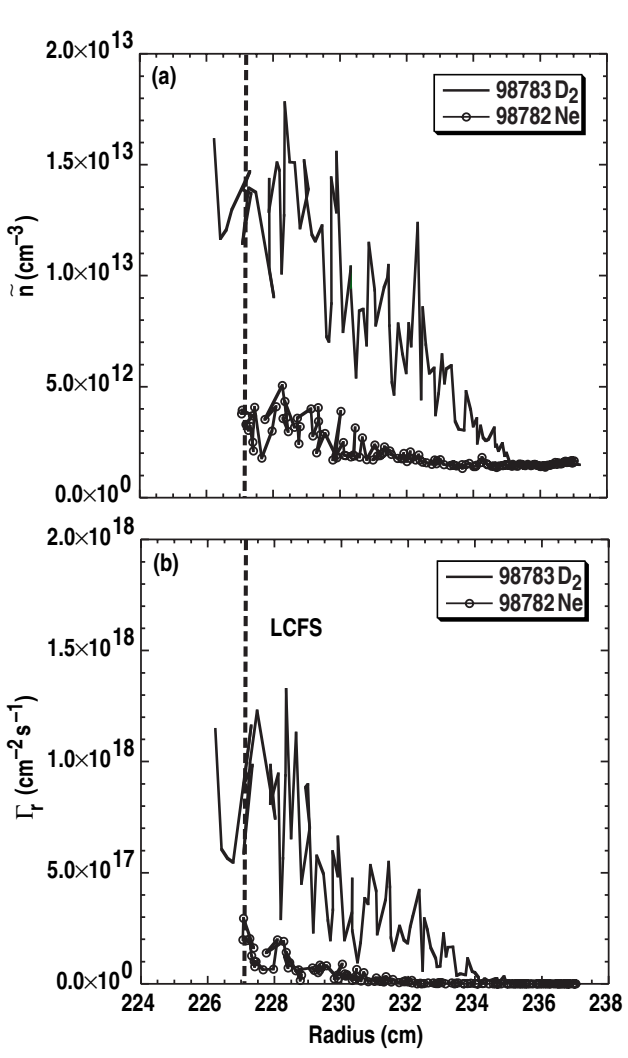

Figure 19. (a) RMS density fluctuation amplitude and (b) particle flux, measured by a fast reciprocating probe, are lower with neon seeding when compared with a discharge with deuterium injection. Probe insertion was over the interval $1.5<t<1.68 \mathrm{~s}$. The discharge conditions are given in the caption to Fig. 16.

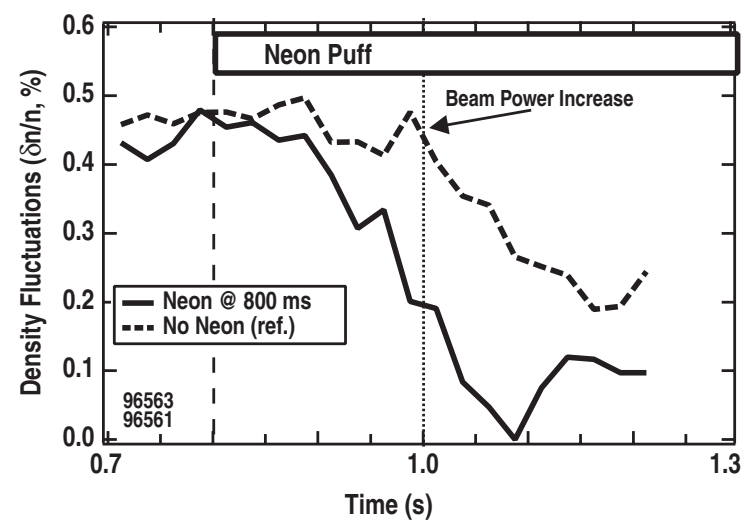

Figure 20. Total density fluctuations at $\rho=0.7$ as a function of time. Solid curves indicate the neon injection discharge (No. 96561) and dashed curves a reference discharge without neon (No. 96563). The discharge parameters were: $I_{p}=1.2 \mathrm{MA}, B_{T}=1.6 \mathrm{~T}$, USN divertor.

3.2.4. Turbulence suppression in impurity seeded L mode diverted discharges We have described the improvements in confinement and decreases in transport which accompanied impurity seeding in DIII-D discharges. Here we discuss turbulence suppression in the mantle region, which is a potential mechanism for explaining this behaviour. The beam emission spectroscopy (BES) diagnostic [28] has measured density fluctuations for L mode discharges with neon injection. In these discharges, the density fluctuation spectra in the 
wavenumber range $0.5 \leqslant k \leqslant 3.0 \mathrm{~cm}^{-1}$ were measured using BES. The temporal response of the fluctuations, integrated over the spectral region $0.1<k_{\perp}<0.6 \mathrm{~cm}^{-1}$, is plotted in Fig. 20 . Shortly after neon injection there is a steady decrease in the fluctuations and the amplitude is significantly lower than that of the reference discharge. Although many discharges exhibit a prompt drop in both fluctuations and thermal diffusivity (e.g., the discharges in Fig. 18), the changes in others, such as the neon seeded discharge shown in Fig. 20, are more gradual. The reasons for these differences have not been completely identified but may be related to the initial impurity puff rate.

The density fluctuation spectrum for the neon discharge shown in Fig. 20 exhibits a decrease, compared with the baseline discharge, above $75 \mathrm{kHz}\left(k \approx 0.4 \mathrm{~cm}^{1}\right)$, and a near complete suppression of fluctuations for $f>200 \mathrm{kHz}$ [29]. The almost complete suppression is observed for wavenumbers $k \rho_{s}>0.4$ at a radial location $\rho \approx 0.7$, where $\rho_{s}=C_{s} / \Omega_{i}$, $C_{s}=\sqrt{T_{e} / m_{i}}$ and $\Omega_{i}=e B / m_{i}$. The radial profile of the neon concentration is highest in the mantle region where BES also shows the largest reduction in density fluctuations. The peak in the neon concentration occurs at $\rho=0.53$ while the maximum reduction observed by BES is at $\rho=0.7$, where the neon concentration is $76 \%$ of its maximum value.

The reduction in density fluctuations with neon seeding shown in Fig. 20 is accompanied by a decrease in ion thermal heat diffusivity, similar to that in the discharge shown in Fig. 18. One mechanism for this reduction is suppression of ion temperature gradient (ITG) modes. Previous studies have shown that if the $\boldsymbol{E} \times \boldsymbol{B}$ shearing rate is larger than the local growth rate then these turbulent modes can be suppressed, leading to a reduction in the thermal diffusivities [29]. Experimentally, neon injection $(Z=10)$ increases the rotational shearing rate in the mantle, allowing an increase in the $\boldsymbol{E} \times \boldsymbol{B}$ shearing rate. Impurity seeding can also lower the ITG growth rates [30]. Gyrokinetic modelling of these discharges shows that these combined effects lead to microturbulence growth rate levels below the $\boldsymbol{E} \times \boldsymbol{B}$ shearing rate, which is consistent with the observed reductions in density fluctuations [29].

3.2.5. Impurities in USN L mode discharges Impurity concentrations and $Z_{\text {eff }}$ are significantly higher in L mode discharges than for the puff and pump scenario presented in Section 2. For example, $Z_{e f f}$ in the two discharges shown in Fig. 16 is $\approx 1.4$ in the baseline case and reaches a maximum of $\approx 3.8$ in the neon seeded case, while the core dilution factor $n_{D} / \bar{n}_{e}$ is 0.9 and 0.65 , respectively. Thus, although a regime of enhanced confinement is achieved, there is a rather large increase in core impurities. Note, however, that even with the higher central impurity density the neutron emission, plotted for the same discharges in Fig. 16(b), is significantly higher than that in the baseline discharge. To date no attempt has been made to minimize the core impurity content of these discharges. The density is well below the Greenwald density limit (Fig. 17), which may contribute to the large impurity content.

\section{Discussion}

In this section we will summarize the results from Sections 2 and 3 and discuss the similarities and differences between the various types of impurity seeded discharge in DIII-D.

\subsection{Puff and pump discharges with impurity seeding}

In puff and pump ELMing $\mathrm{H}$ mode discharges with argon injection, $\gamma_{\text {mantle }}>0.5$ has been obtained with energy confinement times as high as those of ELM-free $\mathrm{H}$ mode, and densities near the Greenwald density limit have been obtained as well as core $Z_{e f f}<2$. Nearly all were limited by the onset of $n=2$ MHD modes, which have been identified as $3 / 2$ tearing modes in cases where fast magnetic data coverage overlapped with the onset of these modes. Steady state discharges with somewhat lower $\gamma_{\text {mantle }}$ and $\bar{n}_{e} / n_{G W}$ and no MHD activity have been obtained, but control of the $3 / 2$ tearing mode remains an important problem in extending these types of discharge to higher densities under steady state conditions. Both ECH current drive and on-axis fast wave current drive are being considered to keep $q(0)$ above 1 , avoiding or delaying the onset of sawteeth.

For puff and pump scenarios we have focused on issues affecting the mantle and core and, in particular, on discharges with a significant increase in mantle radiation. However, as noted earlier, puff and pump discharges with impurity seeding can also have a radiative divertor solution (Fig. 2(b)). Such discharges also exhibit $\mathrm{H}$ mode confinement and there appears to be no clear transition between the radiating mantle and radiating divertor states. This was illustrated in Fig. 13 where impurity puffing was varied over a series of discharges in which divertor radiation was dominant. In these discharges, $W_{\text {ped }}$ was nearly constant and the confinement improvement occurred inside the $\mathrm{H}$ mode transport barrier as the puffing rate increased.

Density in argon seeded puff and pump discharges is higher than for non-seeded discharges, for example Figs 3 and 7. The electron density rise is higher than can be accounted for by the addition of argon ions alone. Direct comparison of discharges with the same deuteron fuelling rate leads us to conclude that particle confinement is improved in impurity seeded discharges. We note that the density pedestal increases, for example Fig. 4(a), and density peaking (not shown) increases in argon injected discharges.

\subsection{L mode discharges}

L mode operation has advantages in eliminating edge instabilities and providing an insulating mantle region, and such discharges have been obtained in DIII-D in both limited and diverted configurations. L mode impurity seeded discharges with enhanced confinement (values of $H_{89 P}$ up to 2) in both divertor and limiter configurations have been obtained in DIII-D. These discharges are evolving, usually with a steady increase in $n_{e}$ and, as in the case of puff and pump discharges, the highest density and confinement are limited by MHD activity. The USN diverted discharges were easier to establish and reproduce, possibly due to lack of MARFE activity. Although there are differences in global confinement scaling between the IWL and USN configurations, there appears to be no fundamental differences in the physical mechanisms producing the improved confinement and higher density. Although the IWL L mode case was not presented, both types of discharge showed a decrease in $\chi_{e f f}$ in a broad 
region and a reduction in the mantle turbulence spectrum measured by BES.

\subsection{Comparison with other tokamak devices with impurity seeding}

The difficulty in achieving steady state radiating mantle discharges in DIII-D is a major difference when compared with TEXTOR RI mode [3] and ASDEX CDH mode [4]. Nearly circular $(\kappa=1.3)$ discharges were achieved in DIII-D, similar to the TEXTOR shape, and while marked confinement improvements are readily observed, they have not been sustained and were limited by either MARFEs or MHD activity. There are several differences between DIII-D and TEXTOR which might account for the observed differences in performance. The TEXTOR RI mode is very sensitive to the horizontal positioning of the plasma and sustained discharges can only be obtained when this has been optimized [3]. Technical constraints presently limit DIII-D limiter operation to the inside wall, although there does appear to be some improvement as the outer gap is decreased. Another difference between these two devices is that TEXTOR quasi-steady-state RI mode discharges generally are obtained at high density, $\bar{n}_{e} / n_{G W} \approx 1$, where higher collisionality can inhibit the onset of tearing modes. Although TEXTOR generally operates with the ALT-II pumped limiter, experiments have been done without pumping, similar to the DIII-D case, and RI mode has been reproduced reliably. For L mode impurity seeded discharges, pumping can allow more effective feedback control and limit disruptions due to radiative collapse, but it is not a fundamental requirement to achieve RI mode.

There are also differences between ASDEX Upgrade CDH mode and DIII-D radiating mantle puff and pump discharges. In DIII-D these discharges are not detached, as reported at ASDEX Upgrade, and DIII-D discharges have type I ELMs [31], not the ASDEX Upgrade type III ELMs. Operation with $\mathrm{H}$ mode confinement near the Greenwald limit for these scenarios is reported at both devices, but as discussed previously steady state operation in DIII-D has not been demonstrated near the Greenwald density limit with impurity seeding.

A general feature of the TEXTOR RI mode is the confinement scaling relation given in Eq. (1). Such a relation does not apply to the DIII-D discharges discussed in this article. Confinement is higher for the same normalized density than that in TEXTOR and we have observed confinement enhancements with neon impurity seeding at lower values of density and radiated power than those reported by the TEXTOR Group. An offset linear scaling relation with a strong dependence on density is apparent in two subsets of USN L mode data (Fig. 17) and confinement is above TEXTOR scaling for IWL L mode discharges (Fig. 15). Clearly the relationship between density and confinement is more complex for DIII-D discharges than for TEXTOR and a scaling relation has not yet been developed. For both $\mathrm{L}$ mode and ELMing $\mathrm{H}$ mode scenarios, confinement improvement is always accompanied by increases in toroidal rotation and rotational shear. Thus momentum input may be an additional parameter required for any DIII-D impurity scaling relation. Nevertheless it is encouraging that DIII-D radiating mantle discharges are above the TEXTOR RI mode scaling relation, suggesting that radiating mantle discharges may scale favourably with machine size.

As noted in Section 1, not all tokamak devices have achieved high density without a degradation in confinement $[8,10]$. In DIII-D L mode discharges, shear suppression of ITG microturbulence is necessary to obtain such conditions, at least in all cases examined to date with the gyrokinetic simulation code. One possibility for the confinement degradation in some tokamaks may be that such devices have not achieved conditions for shear suppression of ITG microturbulence.

\subsection{ITG mode suppression and transport in impurity seeded discharges}

The leading candidate to explain improved confinement in impurity seeded discharges is the suppression of ITG driven turbulence in the mantle region. BES measurements show a clear and reproducible reduction of turbulence with impurity injection into L mode plasmas. Gyrokinetic modelling of these discharges indicates that the introduction of neon raises the threshold for the onset of ITG turbulence, consistent with observed reductions of fluctuations in these plasmas [30]. Similar measurements in ELMing $\mathrm{H}$ mode puff and pump plasmas are difficult due to interference by ELMs and the relatively low levels of turbulence already present. Since there are only small decreases in edge pedestal pressure and $W_{\text {ped }}$ and using the model proposed as a result of work at JET [19], we conclude that the confinement improvement is coming from the core or mantle regions and not from changes in the region of the $\mathrm{H}$ mode transport barrier.

In both the puff and pump and L mode discharges, there is no clear transport barrier associated with impurity seeding. Rather there appears to be a reduction in $\chi_{\text {eff }}$ and $D$ over a large cross-section of the plasma, with the largest reductions typically occurring at $\rho=0.6-0.7$.

We note that the highest densities and highest confinement in both L mode and puff and pump discharges with impurity seeding were obtained after an increase in core toroidal rotation. Increasing rotation was most noticeable at the spontaneous transition in puff and pump discharges and at the onset of impurity puffing in the L mode discharges. Although it is actually $\boldsymbol{E} \times \boldsymbol{B}$ shear suppression which stabilizes microturbulence in the mantle region (Section 3.2.4) of L mode discharges, increasing core rotation always accompanies the higher $\boldsymbol{E} \times \boldsymbol{B}$ shearing rates and thus core rotation is a readily available and useful signature of this process. This signature is also observed in puff and pump scenarios, but more experiments are required to establish a causal relationship with reduced diffusivity and $\boldsymbol{E} \times \boldsymbol{B}$ shear.

\subsection{Impurities}

Fusion reactor and ignition designs have required low $Z_{\text {eff }}$, for example, $Z_{e f f} \leqslant 1.9$ in ITER [32]. This could be a potential problem for the use of non-intrinsic impurities. However, modelling of impurity injection using ITER parameters has produced acceptable solutions with radiated mantle power fractions of $0.7-0.95$ inside the LCFS and only a 5\% degradation in fusion power [33]. In this study krypton 
injection produced $Z_{\text {eff }}(0) \leqslant 1.63$ using several different transport models (neon and argon were somewhat higher). Thus impurity seeding of fusion plasmas with a high radiating power fraction to reduce heat flow to the divertor may be a viable approach in fusion reactors.

Radiating mantle discharges with $Z_{\text {eff }}<2$ in the core region have been obtained in DIII-D high density discharges, for example, Fig. 10, after the spontaneous transition. TRANSP modelling also shows that the thermal neutron flux increases in these discharges after this transition. Core $Z_{\text {eff }}$ is considerably higher in the lower density L mode discharges reported here. Nevertheless, the confinement improvement which accompanies neon injection led to higher neutron emissivity, up to a factor of 2 in Fig. 16. In these experiments, no attempt was made to minimize core $Z_{\text {eff }}$. For example, we expect that increasing the density in L mode discharges will significantly lower core $Z_{\text {eff }}$.

\subsection{SOL characteristics}

As discussed previously, impurity seeding affects both the core and mantle regions. In addition, there are notable changes in the plasma SOL. In puff and pump discharges there are marked decreases in particle and heat fluxes at the outer strike point and in the SOL. This reduction is consistent with a decrease in $P_{L C F S}$. Although these discharges are not detached, the ELM frequency decreases and the energy loss per ELM is quite low, $\triangle W_{E L M} / W_{M H D} \leqslant 0.02$.

Changes in the SOL are also observed in $\mathrm{L}$ mode discharges. Decreases in particle fluxes and density fluctuations measured by a reciprocating probe were shown in Fig. 19 and $\mathrm{D}_{\alpha}$ line intensity steadily decreases after impurity seeding. It is not clear whether these changes in the SOL with impurity seeding create conditions leading to improved particle and energy confinement, or if they are simply a result of changes inside the LCFS.

\subsection{Concluding remarks}

Impurity seeding in DIII-D has produced radiating mantle discharges with improved confinement in a variety of configurations and conditions. ELMing $\mathrm{H}$ mode discharges have produced densities near the Greenwald limit. Lower density L mode neon seeded discharges have shown confinement improvements by as much as a factor of 2 when compared with reference discharges with no impurity injection. Increased confinement and reduced thermal diffusivity in L mode impurity seeded discharges are coincident with a reduction in ITG induced turbulence in the mantle region. Although the fluctuation spectra have not been measured in ELMing $\mathrm{H}$ mode puff and pump discharges, the reduction in thermal diffusivity at the spontaneous transition is qualitatively similar to observed reductions in L mode discharges described in this article.

Normalized confinement in both diverted and inner wall limited L mode discharges is above the RI mode scaling relation, and confinement increases with density. Although no scaling relation has been established for DIII-D radiating mantle discharges, confinement enhancements $H_{93 P}$ are above the TEXTOR RI mode scaling. A DIII-D scaling relation will require more variables, probably including shape parameters and momentum input, than the simple expression in Eq. (1). The application of impurities in fusion reactors will require detailed modelling, but calculations for ITER have demonstrated that solutions are possible with a high radiating fraction inside the LCFS [33]. In such scenarios, feedback control could be used to control impurity radiation but the minimum concentrations required to suppress ITG turbulence have yet to be established.

These experiments show that impurity seeding can be effective in producing high density while maintaining confinement comparable to ELM-free $\mathrm{H}$ mode. The observed decrease in power to the first wall makes the radiating mantle a candidate for reactor scenarios.

\section{Acknowledgements}

This is a report of work supported by the US Department of Energy under Contract Nos DE-AC03-99ER54463, DE-AC0596OR22464, W-7405-ENG-48, DE-AC04-94AL85000, and Grant Nos DE-FG03-95ER54294 and DE-FG02-92ER54139.

\section{References}

[1] Yushmanov, P.N., et al., Nucl. Fusion 30 (1990) 1999.

[2] Lazarus, E.A., et al., Nucl. Fusion 25 (1985) 135.

[3] Messiaen, A.M., et al., Phys. Plasmas 4 (1997) 1690.

[4] Gruber, O., et al., Phys. Rev. Lett. 74 (1995) 4217.

[5] Mori, M., et al., Nucl. Fusion 28 (1988) 1892.

[6] Jackson, G.L., et al., J. Nucl. Mater. 241-243 (1997) 618.

[7] Staebler, G.M., et al., Phys. Rev. Lett. 82 (1999) 1692.

[8] Matthews, G.F., et al., Nucl. Fusion 39 (1999) 19.

[9] Hill, K.W., et al., Nucl. Fusion 39 (1999) 1949.

[10] Kubo, H., et al., Nucl. Fusion 41 (2001) 227.

[11] Winter, J., et al., Phys. Rev. Lett. 71 (1993) 1549.

[12] Ongena, J., et al., in Controlled Fusion and Plasma Physics (Proc. 20th Eur. Conf. Lisbon, 1993), Vol. 17C, Part I, European Physical Society, Geneva (1993) 32.

[13] Unterberg, B., et al., J. Nucl. Mater. 266-299 (1999) 75.

[14] ITER H Mode Database Working Group, Nucl. Fusion 34 (1994) 131.

[15] Greenwald, M., Terry, J.L., Wolfe, S.M., Ejima, S., Nucl. Fusion 28 (1988) 2199.

[16] Wade, M.R., et al., J. Nucl. Mater. 266-299 (1999) 44

[17] Ferron, J.R., Chu, M.S., Jackson, G.L., Phys. Plasmas 7 (2000) 1976.

[18] Groebner, R.J., Osborne, T.H., Phys. Plasmas 4 (1998) 1800.

[19] Fishpool, G.M., Nucl. Fusion 38 (1998) 1373.

[20] Taylor, T.S., Plasma Phys. Control. Fusion 39 (1997) B47.

[21] Goldston, R.J., et al., J. Comput. Phys. 43 (1981) 61.

[22] Burrell, K.H., Phys. Plasmas 4 (1997) 1499.

[23] Hulse, R.A., Nucl. Technol./Fusion 3 (1983) 259.

[24] Hegna, C.C., et al., Phys. Plasmas 1 (1994) 2307.

[25] Goebel, D.M., et al., J. Nucl. Mater. 162-164 (1989) 115.

[26] Johnson, L.C., Hinnov, E., J. Quant. Spectrosc. Radiat. Transf. 13 (1973) 333

[27] Boedo, J.A., Gray, D., Chousal, L., Conn, R., Rev. Sci. Instrum. 69 (1998) 2663.

[28] McKee, G.R., et al., Rev. Sci. Instrum. 70 (1999) 913

[29] McKee, G.R., et al., Phys. Rev. Lett. 84 (2000) 1922.

[30] Murakami, M., et al., Nucl. Fusion 41 (2001) 317.

[31] Doyle, E.J., et al., Phys. Fluids B 3 (1991) 2300.

[32] ITER Physics Basis, Nucl. Fusion 39 (1999) Ch. 1, p. 2137.

[33] Mandrekas, J., Stacey, W.M., Kelly, F.A., Nucl. Fusion 37 (1997) 1015. 